\title{
Using MPI Communication Patterns to Guide Source Code Transformations
}

\author{
Robert Preissl ${ }^{1,2}$, Martin Schulz ${ }^{1}$, Dieter Kranzlmüller ${ }^{2}$, \\ Bronis R. de Supinski ${ }^{1}$, and Daniel J. Quinlan ${ }^{1}$ \\ ${ }^{1}$ CASC, Lawrence Livermore National Laboratory, USA* \\ \{preissl2, schulzm, bronis, dquinlan\}@llnl.gov \\ ${ }^{2}$ GUP, Johannes Kepler University Linz, Austria/Europe \\ $\{$ rpreissl, dk\}@gup.jku.at
}

\begin{abstract}
Optimizing the performance of HPC software requires a highlevel understanding of communication patterns as well as their relation to source code structures. We describe an algorithm to detect communication patterns in parallel traces and show how these patterns can guide static code analysis. First, we detect patterns that identify potential bottlenecks in MPI communication traces. Next, we associate the patterns with the corresponding nodes in an abstract syntaxtree using the ROSE compiler framework. Finally we perform static analysis on the annotated control flow and system dependence graphs to guide transformations such as code motion or the automatic introduction of MPI collectives.
\end{abstract}

\section{Introduction}

With today's increasingly complex and highly scalable parallel systems, we require approaches that combine static (compile time) and dynamic (run time) information to capture sufficient information to optimize their applications. Similar techniques already support feedback guided compilation; however, these approaches limit the kind of data they collect and the optimizations that they apply (often restricted to simple localized decisions like inlining or code layout).

In this paper, we apply this principle to the analysis and optimization of global communication behavior, which is a primary source of inefficiency in parallel machines 2]. We use a suffix tree algorithm to detect repeating patterns in communication traces and map the patterns to static data structures. For the latter part we rely on ROSE, a comprehensive and flexible toolkit for the generation of source-to-source translators. We present early experiences on static source transformations exploiting the additional runtime information.

Sec. 2 gives an overview of the approach and shows the most important steps of the process. In Sec. 3 we formally define the repeating structures in strings and then compare the runtime behavior and memory usage of two different

\footnotetext{
* Part of this work was performed under the auspices of the U.S. Department of Energy by Lawrence Livermore National Laboratory under Contract DE-AC52-07NA27344. (LLNL-CONF-400356).
}

M. Bubak et al. (Eds.): ICCS 2008, Part III, LNCS 5103, pp. 253-260, 2008.

(C) Springer-Verlag Berlin Heidelberg 2008 


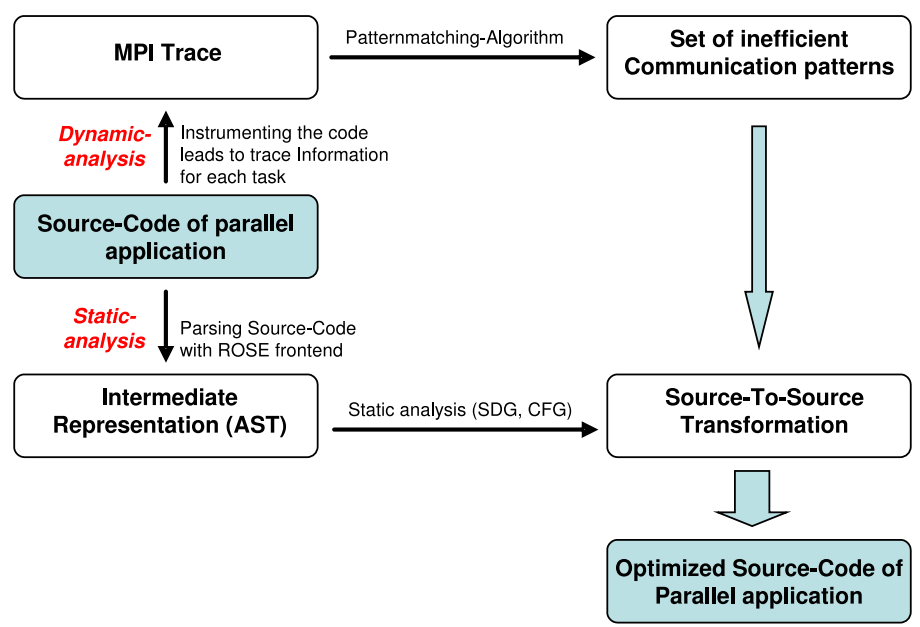

Fig. 1. Flow diagram of the main approach

algorithms that use the structures to find MPI communication patterns. Sec. 4 gives an overview of the ROSE compiler infrastructure that enables us to perform static analysis of the associated regions in the parallel code and to carry out source-to-source transformations. Sec. 5 provides a concrete example of a successfully transformed parallel code that achieves better performance by overlapping communication and computation. In the final sections we discuss aspects of future research and present our conclusions.

\section{Combing Static and Dynamic Analysis}

The analysis of MPI communication patterns has traditionally been used for high-level understanding and error detection 345. In this work we extend the use of patterns to static analysis and source code transformations for performance optimization. We detect repetitive patterns of inefficient communication at runtime (e.g., poorly implemented broadcast operations) and use this information to optimize these heavily used structures in existing applications statically by replacing them with more efficient equivalent operations.

Fig. 1 gives an overview of our approach: we instrument the target MPI application, generate an MPI trace of the program executed under a given set of parameters, and then use pattern matching to isolate recurring inefficient communication structures. We also generate an abstract syntax tree (AST) of the application and perform a static analyses to extract control and data flow. We then map the detected patterns onto this information and use these annotations to guide potential source-to-source transformations.

This process comes with four major challenges:

1. Detecting MPI operations that would make recurring patterns interesting

2. Finding and filtering the patterns that involve these operations 
3. Determining if the optimizations applicable to the patterns can be applied safely to the AST (possibly through specialization)

4. Transforming the related source code fragments

\section{Detecting Patterns in MPI Communication Traces}

MPI communication patterns are repeating communication structures in an MPI event graph [6]. First each MPI event is encoded into a 32 bit integer, such that each task's trace information is represented by an array of integer variables. For each task, we compute all repetitive and maximal repetitive sequences [7].

Definition 1. A maximal pair of a string $S$ of length $n$ is a triple $(p 1, p 2, l)$, such that

$$
\begin{gathered}
S\left[p_{1}, p_{1}+l-1\right]=S\left[p_{2}, p_{2}+l-1\right], \text { but } \\
S\left[p_{1}-1\right] \neq S\left[p_{2}-1\right] \text { and } S\left[p_{1}+l\right] \neq S\left[p_{2}+l\right]
\end{gathered}
$$

where $p_{1}, p_{2}$ denote the starting positions of the two substrings and $l$ gives their lengths. A maximal repeat is a string represented by such a triple.

We use the maximal repeats, which are a subset of all repeated sequences, to select a start sequence. Starting from a maximal repeat, we identify global communication patterns that they contain. Using maximal repeats is crucial for finding repeating communication patterns efficiently. We have currently implemented two ways to detect repetitive structures in MPI traces: a naive algorithm based on convolution and a more advanced technique using suffix trees.

Our naive convolution method aligns the left end of the pattern $P$ with the left end of the string $S$ and then compares the characters of $P$ and $S$ left to right until either two unequal characters are found or until $P$ is exhausted. It then shifts $P$ to the right one place and repeats the comparisons process. If $n$ is the length of $P$ and $m$ is the length of $S$, this approach makes $\Theta(n m)$ [7] comparisons in the worst case. Since there is possibly a huge number of MPI events in the trace, this method take significant runtime.

Our more advanced compressed suffix tree approach borrows ideas from computational biology for finding repeating gene structures in huge DNA sequences [7. The suffix tree for a string $S$ with length $m$ is a rooted directed tree with exactly $m$ leaves and whose labels correspond to substrings of $S$. For any leaf $i$, the concatenation of the edge-labels on the path from the root to leaf $i$ exactly spells out the suffix of $S$ starting at position $i$.

Definition 2. An internal node $v$ of the suffix tree for string $S$ is called left diverse if at least two leaves with suffix position $i$ and $j$ in $v^{\prime}$ s subtree have different left characters, that is, $S[i-1] \neq S[j-1]$

Theorem 1 (D. Gusfield). The substring $\alpha$ labeling the path to an internal node $v$ of the suffix tree for string $S$ is a maximal repeat iff $v$ is left diverse. 


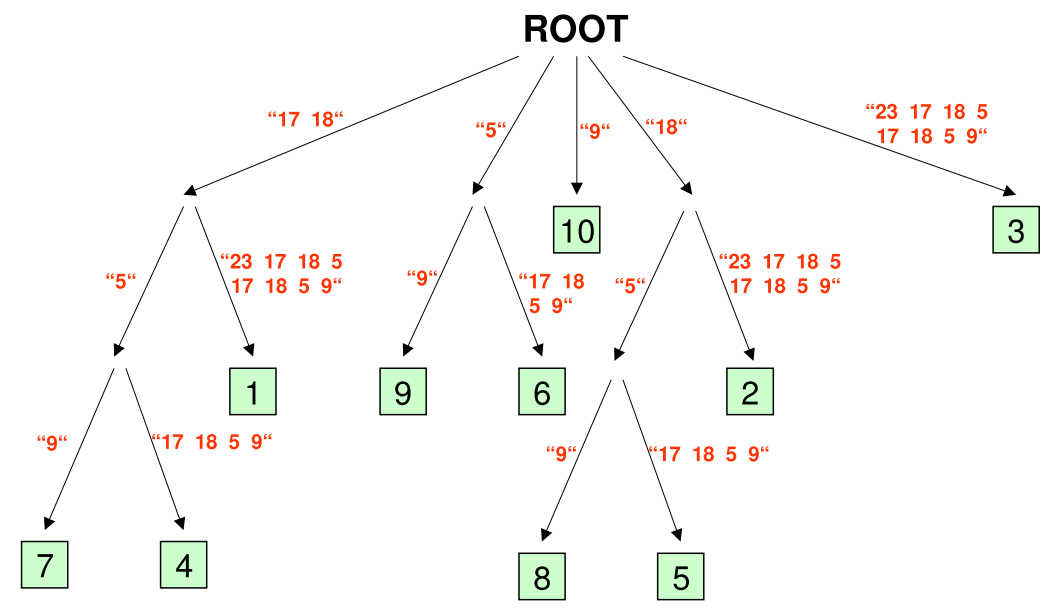

Fig. 2. Suffix for a string representing MPI events of a trace

D. Gusfield describes algorithms (e.g., Ukkonen's algorithm) for constructing such trees with all its suffix links for a string $S$ with length $m$ in $\Theta(m)$ time [7. However, these algorithms are not space efficient, particularly with large alphabets, as with MPI traces. Thus, we use an alternative approach. The compressed suffix tree [8] for a string $S$ with length $m$ of an alphabet $\Sigma$ occupies $\Theta(m \log |\Sigma|)$ bits. The final time requirement for creating the tree is $\Theta(m \log |m| \log |\Sigma|)$, being reasonably close to the best current theoretical result 9 .

Fig. 2 shows a suffix tree of the string $S=$ "17, 18, 23, 17, 18, 5, 17, 18, 5, 9", in which each integer represents an MPI event. We can easily see from the suffix tree that $\langle 17,18\rangle$ with starting positions $(1,4$ and 7$)$ and $\langle 17,18,5\rangle$ with starting positions (4 and 7 ) are maximal repeats. In addition to these (maximal) repeats, $S$ has the repeat $\langle 18,5\rangle$ with starting positions (5 and 8 ).

We must choose a repeat on a specific task from which to start the pattern matching process. Since each task typically has many maximal repeats, the time required to compute a communication pattern for all maximal repeats is generally prohibitive. Hence, we first filter start-repeats and then compute only communication patterns that involve these filtered events. This filter step is either guided by special seed events, e.g. events in the trace with a huge difference between start- and end-time, or by focusing on selected MPI operations.

Fig. 3 shows an example MPI trace in which the events in bold highlight a repeating set of MPI operations that our pattern-detection algorithm finds. This pattern is potentially equivalent to a broadcast operation using a tree-based communication structure. In this example, our static analysis must verify that the pattern is a broadcast operation and, if so, that we can replace the associated code segments with an equivalent, but probably more efficient version in the form of a native MPI collective. This is promising because it will enable programmers, that do not have a broad knowledge of MPI to apply more efficient functions defined by the MPI standard in without having to use them explicitly. 


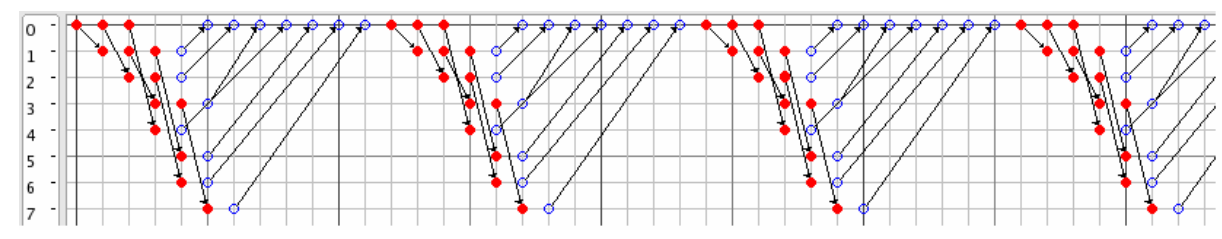

Fig. 3. A detected pattern representing a broadcast operation in an MPI trace

\section{Static Analysis with ROSE}

A communication pattern extracted from the runtime trace only points to a potential bottleneck. Further, the pattern is initially only valid for the particular input set used during the application run. We must verify the pattern occurs across all control flows and investigate pattern specific global data flow constraints (e.g., in the case of a suspected broadcasts that the same data is communicated in all messages). The required information to achieve this goal is encapsulated in the System-Dependence-Graph (SDG) [10] and the ControlFlow-Graph (CFG).

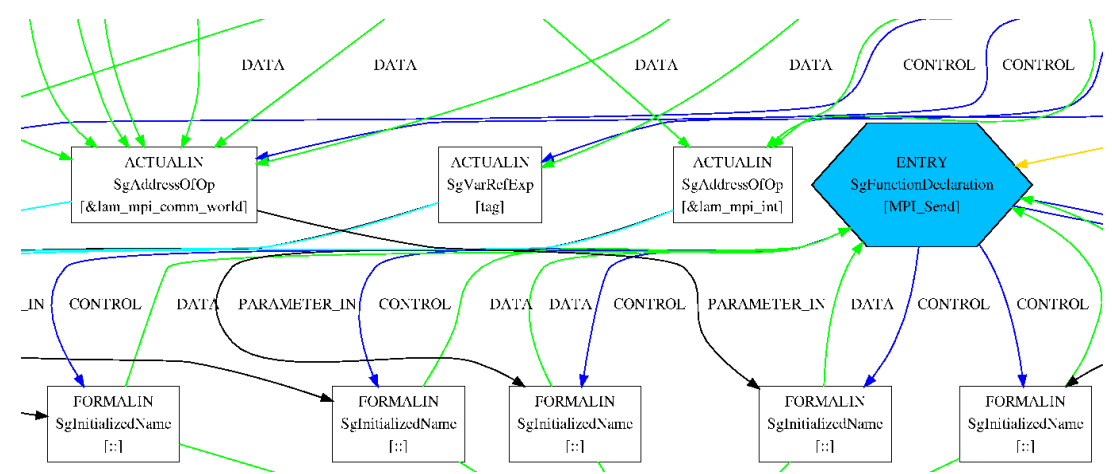

Fig. 4. Excerpt of the System-Dependence-Graph (SDG) of a parallel program

We use the ROSE open-source compiler infrastructure to generate both of these graphs. ROSE is a tool kit to generate custom source-to-source translators. It provides mechanisms to translate input source code into an intermediate representation (AST) [11, libraries to traverse and manipulate the information stored in the AST, as well as mechanisms to transform the changed AST information back into valid source code. The representation within the AST as well as the supporting data structures is powerful enough to readily exploit knowledge of the architecture, parallel communication characteristics, and cache architecture in the specification of the transformations [12.

Fig.4illustrates a small excerpt of an SDG generated by ROSE providing both data and control dependency information. The specific example shows the nodes 


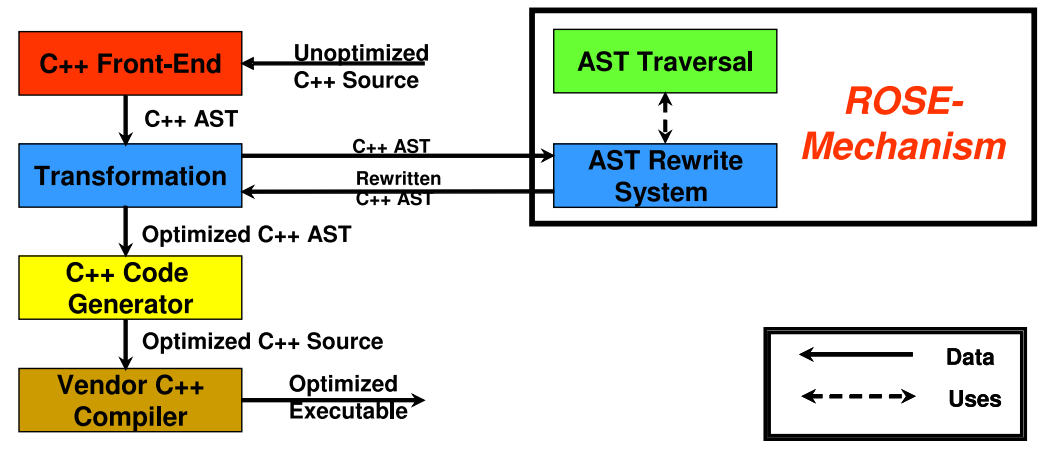

Fig. 5. The ROSE compiler infrastructure

and data-dependence egdes that represent flow of data between statements or expressions as well as control-dependence edges that represent control conditions on which the execution of a statement or expression depends, in this case around an MPI_Send function in a parallel application.

The flow diagram in Fig. 5 reflects the complete approach, transforming unoptimized $\mathrm{C}++$ code based on user defined abstractions into highly optimized code. In our approach we have defined specific transformations to MPI code structures based on dynamic analysis results in the form of inefficient MPI communication patterns.

\section{Examples and Early Results}

The following simple example illustrates how we can use our techniques to replace blocking with non-blocking communication to overlap communication and computation for better overall performance. Fig. [6] shows the structure of the corresponding "Late-Sender" pattern: the receiving task wastes useful time in waiting for a message to be sent by another task. We detect this pattern from our communication trace and then apply a source code transformation using the ROSE

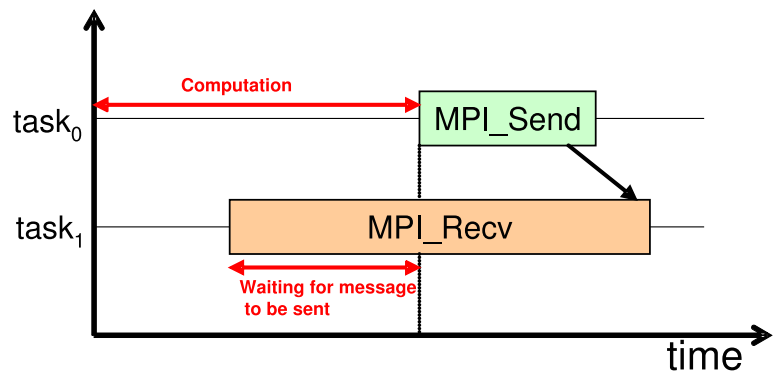

Fig. 6. Late-Sender pattern 


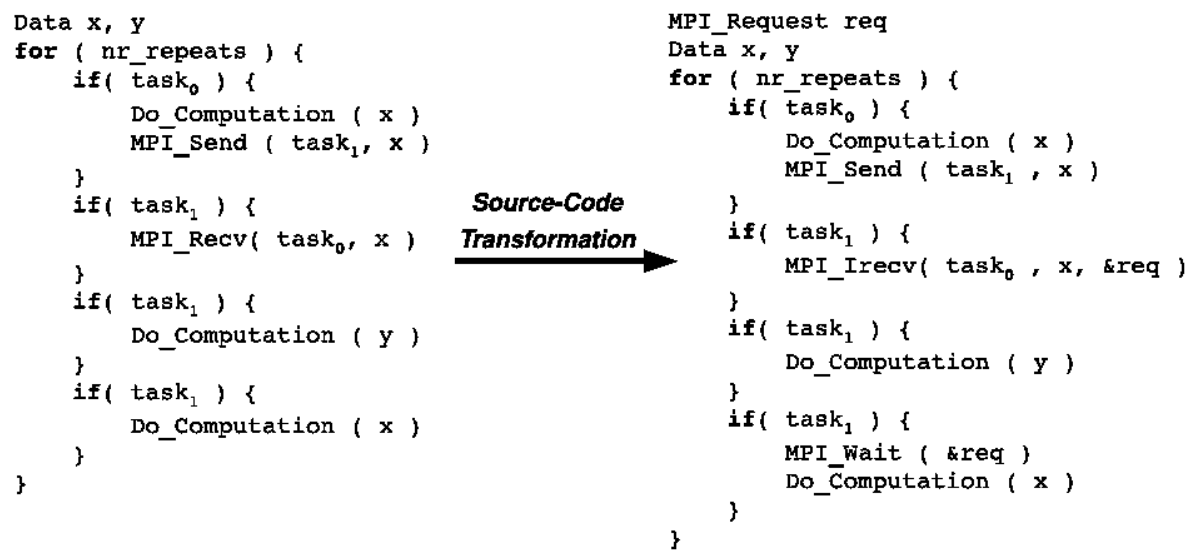

Fig. 7. Source code transformation: before (left), after (right)

compiler framework that introduces non-blocking communication, as shown in the pseudo-code in Fig. 7. Our transformation replaces the blocking MPI_Recv with its non-blocking MPI_Irecv version and adds the matching MPI_Wait so as to provide the largest possible communication/computation overlap allowed by data dependencies on the receive buffer.

\section{Conclusion and Future Work}

This paper presents our work to automate the analysis and optimization of parallel scientific applications by combining dynamic runtime information in the form of communication patterns with static analysis and code transformation. We use the dynamic information to identify the Send- and Receive Events that are part of inefficient communication patterns and are good targets for source code optimizations.

In our ongoing research, we are extending the library of inefficient MPI communication patterns that we optimize beyond the communication/computation overlap transformation described here. In particular, we will introduce static and dynamic analysis as well as the corresponding transformation engine for automatically adding collective operations. Further, we will provide several other novel methods for selecting and filtering MPI seed events that will help us find more complex patterns that lead to communication bottlenecks.

\section{References}

1. The List of Worlds 500 Fastest Supercomputers, www.top500.org

2. Bassetti, F., Davis, K., Quinlan, D.: Improving Scalability with Loop Transformations and Message Aggregation in Parallel Object-Oriented Frameworks for Scientific Computing. In: Computing, Information, and Communications Division, Los Alamos, NM, USA (1998) 
3. Kranzlmüller, D., Grabner, S., Volkert, J.: Event Graph Visualization for Debugging Large Applications. In: Proc. of SIGMETRICS Symposium on Parallel and Distributed Tools, SPDT Philadelphia, PA, USA, pp. 108-117 (1996)

4. Kranzlmüller, D.: Communication Pattern Analysis in Parallel and Distributed Programs. In: Proc. of the 20th IASTED International Multi-Conference Applied Informatics (AI 2002), International Symposia on Software Engineering, Databases, and Applications, International Association of Science and Technology for Development (IASTED). ACTA Press, Innsbruck (2002)

5. Knüpfer, A., Kranzlmüller, D., Nagel, W.E.: Detection of Collective MPI Operation Patterns. In: Kranzlmüller, D., Kacsuk, P., Dongarra, J. (eds.) EuroPVM/MPI 2004. LNCS, vol. 3241, pp. 259-267. Springer, Heidelberg (2004)

6. Kranzlmüller, D.: Event Graph Analysis for Debugging Massively Parallel Programs. PhD thesis, GUP Linz, Johannes Kepler University Linz, Austria (2000)

7. Gusfield, D.: Algorithms on Strings, Trees, and Sequences. Computer Science and Computational Biology (1997)

8. Dixit, K., Gerlach, W., Maekinen, Vaelimaeki, N.: Engineering a Compressed Suffix Tree Implementation. Department of Computer Science, Series of Publications C, Report C-2006-37, University of Helsinki, Finland (2006)

9. Hon, W.-K., Sadakane, K., Sung, W.-K.: Breaking a time-and-space barrier in constructing full-text indices. In: FOCS 2003: Proceedings of the 44th Annual IEEE Symposium on Foundations of Computer science, Washington, DC, USA, p. $251(2003)$

10. Horwitz, S., Reps, T., Binkley, D.: Interprocedural slicing using dependence graphs. ACM Transactions on Programming Languages and Systems, p. 26 (1990)

11. Schordan, M., Quinlan, D.: A source-To-Source Architecture for User-Defined Optimizations. Lawrence Livermore National Laboratory, USA (2003)

12. Quinlan, D.: Compiler Support for Object-Oriented Frameworks. Lawrence Livermore National Laboratory, USA (1999)

13. Panas, T., Quinlan, D., Vuduc, R.: Tool Support for Inspecting the Code Quality of HPC Architectures. Lawrence Livermore National Laboratory, USA (2007) 